\title{
PLANEJAMENTO EXPERIMENTAL APLICADO À PURIFICAÇÃO DE GLICEROL UTILIZANDO DIFERENTES ADSORVENTES
}

\author{
G. N. SCHUABB ${ }^{1}$, A. P. ALVES ${ }^{1}$ e M. F. MENDES ${ }^{1}$ \\ ${ }^{1}$ Universidade Federal Rural do Rio de Janeiro, Departamento de Engenharia Química \\ Laboratório de Termodinâmica Aplicada e Biocombustíveis \\ E-mail para contato: marisamf@ufrri.br
}

\begin{abstract}
RESUMO - O aumento na produção de biodiesel nos últimos anos, dada a crescente demanda energética e necessidade do uso de fontes alternativas de energia em detrimento ao uso de combustível fóssil, tem atraído a necessidade do desenvolvimento de tecnologias para purificação do glicerol, principal subproduto gerado pela reação transesterificação de óleos vegetais e gordura animal. Entre os vários processos de purificação já existentes no mercado, a adsorção é uma técnica promissora na remoção de contaminantes do glicerol, dado ao seu baixo custo e simplicidade operacional. Nesse contexto, o presente estudo propõe a otimização do processo de purificação do glicerol, através de um planejamento experimental, utilizando três diferentes adsorventes (argila clarificante Pure-Flo Supreme B81, terra diatomácea e carvão ativado) e uma solução sintética de glicerina P.A. e óleo de soja comercial, simulando o glicerol bruto contaminado. Os resultados obtidos foram satisfatórios quando ao percentual de remoção de contaminantes.
\end{abstract}

\section{INTRODUÇÃO}

Nos últimos anos, com as mudanças climáticas e a crise energética causada pelo aumento no preço do petróleo e esgotamento dos combustíveis fósseis, houve a necessidade da busca de novas fontes de energia. Nesse contexto, o biodiesel vem se tornando uma alternativa viável para substituição dos combustíveis fósseis, resultado de incentivos políticos e governamentais, e é considerado promissor no mundo todo, por isso, sua produção tem crescido anualmente (DASARI et al., 2005).

O biodiesel é produzido pela reação de transesterificação de óleos vegetais ou gorduras animais e a cada 3 mols de biodiesel produzidos, 1 mol é do subproduto glicerol, correspondendo a $10 \%$ do volume total da produção de biodiesel (SILVA et al., 2009) e, com isso, o aumento projetado para produção de biodiesel elevará também a quantidade do subproduto glicerol no mercado. Esse subproduto contém muitas impurezas inerentes à produção e a sua purificação aumenta seu valor agregado, ajudando na economia e viabilidade da produção de biodiesel, criando também uma alternativa para reduzir seu excesso (ANITHA et al., 2016). No entanto, a purificação é um processo dispendioso, sendo necessários novos métodos de purificação mais baratos e eficientes. 
Dentre os vários métodos de purificação, que incluem destilação, purificação por membrana, troca iônica e extração química (CHUN-HUI et al., 2008; SDRULA, 2010), a adsorção tem se mostrado uma técnica promissora e eficiente para remoção dos contaminantes do glicerol e possui vantagens sobre as outras operações de separação como simplicidade de projeto e operação, seletividade dos adsorventes e baixo custo. Diante do exposto, o objetivo deste trabalho é avaliar a adsorção como processo de purificação de uma solução sintética de glicerina P.A. contaminada com óleo de soja, simulando uma carga de glicerol bruto, comparando a eficiência de diferentes adsorventes, a partir de um planejamento experimental.

\section{MATERIAIS E MÉTODOS}

\subsection{Materiais}

Para os experimentos de adsorção foram utilizados os seguintes adsorventes: carvão ativado (CA, Vetec Química Ltda.), argila clarificante Pure Flo Supreme B81 (AC, Oil-Dri. Chicago, EUA) e terra diatomácea (TD). Para o preparo da solução sintética foi utilizado óleo de soja comercial e glicerina (Vetec Química Ltda.) nas proporções de 30, 50 e 70\% v/v.

\subsection{Procedimento Experimental}

Testes com glicerina P.A. e óleo de soja foram realizados com o intuito de verificar a influência da concentração inicial de glicerina na solução e a ocorrência de competição entre a glicerina e os contaminantes no processo de purificação por adsorção. Para isso, foi utilizado um planejamento experimental $\left(2^{3}\right)$, com 8 ensaios e 3 pontos centrais, totalizando 11 experimentos, para os 3 adsorventes em separado, com o intuito de avaliar a eficiência dos adsorventes e comparação entre eles. As variáveis independentes analisadas estatisticamente foram: temperatura, concentração inicial de glicerol e quantidade de adsorvente, conforme mostrado na Tabela 1. A variável dependente foi a porcentagem de glicerol final de cada ensaio (ALVES, 2017).

O procedimento experimental consistiu na utilização de erlenmeyers de $125 \mathrm{~mL}$, cada um contendo a quantidade de material adsorvente e $30 \mathrm{~g}$ de solução sintética de glicerina/óleo de soja, colocados no shaker à temperatura de interesse e agitação de $250 \mathrm{rpm}$. As alíquotas foram retiradas no tempo pré-determinado de $30 \mathrm{~min}$ e o conteúdo de cada erlenmeyer foi filtrado para remoção dos adsorventes. As amostras foram avaliadas quanto ao percentual de remoção de impurezas, utilizando espectrofotometria na região do UV/visível, seguindo a norma ASTM E169-04.

Tabela 1 - Variáveis e níveis estudados no planejamento $2^{3}$.

\begin{tabular}{|c|c|c|c|}
\hline \multirow{2}{*}{ Variáveis } & \multicolumn{3}{|c|}{ Níveis } \\
\cline { 2 - 4 } & Inferior $(-)$ & Central $(0)$ & Superior $(+)$ \\
\hline Temperatura $\left({ }^{\circ} \mathrm{C}\right)$ & 25 & 32,5 & 40 \\
\hline Quantidade de adsorvente $(\mathrm{QA}, \mathrm{g})$ & 1,2 & 1,8 & 2,4 \\
\hline Porcentagem de glicerol $(\%)$ & 30 & 50 & 70 \\
\hline
\end{tabular}




\section{RESULTADOS E DISCUSSÕES}

A Tabela 2 apresenta os resultados de concentração de glicerol (variável dependente), para todos os experimentos realizados. Nota-se que a concentração final de glicerol variou entre 29,59 e $97,25 \%$.

Tabela 2 - Resultados do processo de adsorção para purificação do glicerol.

\begin{tabular}{|c|c|c|c|c|c|c|}
\cline { 5 - 7 } & \multicolumn{2}{|c|}{ T $\left({ }^{\circ} \mathrm{C}\right)$} & \multirow{2}{*}{$\mathrm{CG}(\%)$} & \multirow{2}{*}{ QA $(\mathrm{g})$} & \multicolumn{3}{c|}{ \% Glicerol } \\
\cline { 5 - 7 } & & & & $\mathrm{AC}$ & $\mathrm{TD}$ & $\mathrm{CA}$ \\
\hline 1 & 25 & 30 & 1,2 & 33,78 & 30,73 & 29,59 \\
\hline 2 & 40 & 30 & 1,2 & 32,17 & 32,11 & 81,96 \\
\hline 3 & 25 & 70 & 1,2 & 89,77 & 90,24 & 72,92 \\
\hline 4 & 40 & 70 & 1,2 & 86,12 & 78,43 & 95,39 \\
\hline 5 & 25 & 30 & 2,4 & 36,36 & 97,25 & 32,36 \\
\hline 6 & 40 & 30 & 2,4 & 36,98 & 35,00 & 91,30 \\
\hline 7 & 25 & 70 & 2,4 & 90,26 & 87,57 & 95,59 \\
\hline 8 & 40 & 70 & 2,4 & 89,46 & 87,53 & 70,00 \\
\hline 9 & 32,5 & 50 & 1,8 & 57,09 & 50,41 & 49,09 \\
\hline 10 & 32,5 & 50 & 1,8 & 57,58 & 51,15 & 49,34 \\
\hline 11 & 32,5 & 50 & 1,8 & 57,80 & 51,81 & 50,39 \\
\hline
\end{tabular}

O maior teor de glicerol foi obtido com uma concentração inicial de $70 \%$, indicando que a concentração de glicerol é o fator mais importante no processo de adsorção.

A concentração final de glicerol utilizando terra diatomácea como adsorvente variou entre $97,25 \%$ e $30,73 \%$. O melhor ponto operacional foi de $25{ }^{\circ} \mathrm{C}$, usando $2,4 \mathrm{~g}$ de adsorvente e concentração inicial de glicerol de 30\%. Para o carvão ativado, a concentração final de glicerol variou entre 29,59 e $95,59 \%$. A melhor condição operacional para este adsorvente foi a $25{ }^{\circ} \mathrm{C}$, concentração inicial de $70 \%$ glicerol e $2,4 \mathrm{~g}$ de adsorvente. $\mathrm{O}$ percentual de remoção de óleo de soja nessas condições foi de 89,25\%. O mesmo comportamento foi observado utilizando argila clarificante. Nese caso, o pior resultado foi o experimento 1 , onde houve a remoção de glicerol ao invés do óleo de soja contaminante, sendo essa remoção de $0,588 \%$ de glicerol.

A influência das três variáveis independentes na purificação de glicerol foi analisada estatisticamente e para que os efeitos calculados sejam estatisticamente significativos, o valor de p correspondente deve ser menor ou igual a 0,05 para o nível de confiança estudado (95\%). Os coeficientes de regressão, erro padrão, t-valor e p-valor foram calculados em função do teor de glicerol final e estão listados na Tabela 3. De acordo com o p-valor presente na Tabela 3 , conclui-se que todas as variáveis são significativas ( $\mathrm{p}$-valor $<0,05$ ) para os resultados da purificação de glicerol. Além disso, pode-se observar a partir do teste $\mathrm{F}$ que a análise de 
regressão também foi significativa para todos os adsorventes, uma vez que o valor do $\mathrm{F}_{\text {calculado }}$ foi maior do que o $\mathrm{F}_{\text {tabelado. }}$

Como o $\mathrm{F}_{\text {cal }}$ foi maior que $\mathrm{F}_{\mathrm{tab}}$, para todos os adsorventes, com base na análise de variância e coeficientes de regressão, um modelo de regressão foi construído a partir das variáveis significativas. As equações 1, 2 e 3 representam o modelo codificado que descreve as superfícies de resposta para o teor de glicerol purificado com argila clarificante, terra diatomácea e carvão ativado, respectivamente.

$$
\begin{aligned}
& Y=-7,95875-0,2005 \mathrm{x}_{1}+1,51246 \mathrm{x}_{2}-0,39444 \mathrm{x}_{3}-0,00288 \mathrm{x}_{1} \mathrm{x}_{2}+0,14111 \mathrm{x}_{1} \mathrm{x}_{3}-0,03708 \\
& \mathrm{x}_{2} \mathrm{x}_{3}
\end{aligned}
$$

$Y=-49,5175-0,6615 \mathrm{x}_{1}+0,7825 \mathrm{x}_{2}+95,4201 \mathrm{x}_{3}+0,0408 \mathrm{x}_{1} \mathrm{x}_{2}-1,4406 \mathrm{x}_{1} \mathrm{x}_{3}-0,6560 \mathrm{x}_{2} \mathrm{x}_{3}$

$Y=-263,980+8,646 x_{1}+3,994 x_{2}+47,136 x_{3}-0,095 x_{1} x_{2}-1,152 x_{1} x_{3}-0,154 x_{2} x_{3}$

sendo $\mathrm{Y}$ a concentração final de glicerol, $\mathrm{x}_{1}, \mathrm{x}_{2} \mathrm{e} \mathrm{x}_{3}$ correspondem a temperatura, quantidade inicial de glicerol e quantidade de adsorvente, respectivamente. Os coeficientes de correlação entre os modelos e os resultados experimentais foram de 3,24\%, 18,28\% e 17,75\% para a argila clarificante, terra diatomácea e carvão ativado, respectivamente, demostrando que para os três adsorventes os modelos possuem boas correlações (ALVES, 2017).

Tabela 3 - Análise do ANOVA para os experimentos com cada adsorvente.

\begin{tabular}{|c|c|c|c|c|}
\hline Fatores & $\begin{array}{c}\text { Coeficiente de } \\
\text { regressão }\end{array}$ & $\begin{array}{c}\text { Erro } \\
\text { padrão }\end{array}$ & T-valor & P-valor \\
\hline \multicolumn{5}{|c|}{ Argila clarificante } \\
\hline Média & $-7,95875$ & 2,496249 & 553,6291 & 0,000003 \\
\hline \multicolumn{5}{|c|}{ Coeficiente linear } \\
\hline Temperatura $\left(\mathrm{X}_{1}\right)$ & $-0,20050$ & 0,069067 & $-5,2918$ & 0,033905 \\
\hline Concentração de Glicerol $\left(\mathrm{X}_{2}\right)$ & 1,51246 & 0,034467 & 210,4264 & 0,000023 \\
\hline Quantidade de adsorvente $\left(\mathrm{X}_{3}\right)$ & $-0,39444$ & 1,092631 & 10,9143 & 0,008290 \\
\hline \multicolumn{5}{|c|}{ Coeficientes de interação } \\
\hline $\begin{array}{c}\text { Temperatura e Quantidade de } \\
\text { adsorvente }\end{array}$ & 0,14111 & 0,028556 & 4,9416 & 0,038596 \\
\hline Temperatura e concentração de glicerol & $-0,00288$ & 0,000857 & $-3,36573$ & 0,078077 \\
\hline Quantidade de adsorvente e & $-0,03708$ & 0,010708 & $-3,4630$ & 0,074222 \\
\hline concentração de glicerol & Terra diatomácea \\
\hline Média & $-49,5175$ & 4,810284 & 298,0024 & 0,000011 \\
\hline \multicolumn{7}{|c|}{ Coeficiente linear } \\
\hline Temperatura $\left(\mathrm{X}_{1}\right)$ & $-0,6615$ & 0,133093 & $-36,7092$ & 0,000741 \\
\hline Concentração de Glicerol $\left(\mathrm{X}_{2}\right)$ & 0,7825 & 0,066418 & 75,0539 & 0,000177 \\
\hline Quantidade de adsorvente $\left(\mathrm{X}_{3}\right)$ & 95,4201 & 2,105506 & 38,2842 & 0,000682 \\
\hline \multicolumn{7}{|c|}{ Coeficientes de interação } \\
\hline Temperatura e Quantidade de & $-1,4406$ & 0,055027 & $-26,1790$ & 0,001456 \\
\hline
\end{tabular}




\begin{tabular}{|c|c|c|c|c|}
\hline adsorvente & & & & \\
\hline Temperatura e concentração de glicerol & 0,0408 & 0,001651 & 24,7454 & 0,001629 \\
\hline $\begin{array}{c}\text { Quantidade de adsorvente e } \\
\text { concentração de glicerol }\end{array}$ & $-0,6560$ & 0,020635 & $-31,7924$ & 0,000988 \\
\hline \multicolumn{5}{|c|}{ Carvão ativado } \\
\hline Média & $-263,980$ & 4,737660 & 313,8039 & 0,000010 \\
\hline \multicolumn{5}{|c|}{ Coeficiente linear } \\
\hline Temperatura $\left(\mathrm{X}_{1}\right)$ & 8,646 & 0,131083 & 55,4517 & 0,000325 \\
\hline Concentração de Glicerol $\left(\mathrm{X}_{2}\right)$ & 3,994 & 0,065416 & 50,5826 & 0,000391 \\
\hline Quantidade de adsorvente $\left(\mathrm{X}_{3}\right)$ & 47,136 & 2,073718 & 4,8127 & 0,040564 \\
\hline \multicolumn{5}{|c|}{ Coeficientes de interação } \\
\hline $\begin{array}{c}\text { Temperatura e Quantidade de } \\
\text { adsorvente } \\
\end{array}$ & $-1,152$ & 0,054196 & $-21,2653$ & 0,002204 \\
\hline Temperatura e concentração de glicerol & $-0,095$ & 0,001626 & $-58,6499$ & 0,000291 \\
\hline $\begin{array}{l}\text { Quantidade de adsorvente e } \\
\text { concentração de glicerol }\end{array}$ & $-0,154$ & 0,020324 & $-7,6010$ & 0,000687 \\
\hline
\end{tabular}

Foram geradas superfícies de resposta para a definição das condições mais apropriadas para maximização da variável dependente. A Figura 1 ilustra as superfícies de resposta que ilustram a purificação de glicerol em função da temperatura, da concentração inicial de glicerol e da quantidade de adsorvente.

Figura 1 - Variação da concentração final de glicerol em função da (a) temperatura e concentração inicial de glicerol, (b) concentração de glicerol e quantidade de terra diatomácea e (c) temperatura e quantidade de carvão ativado.
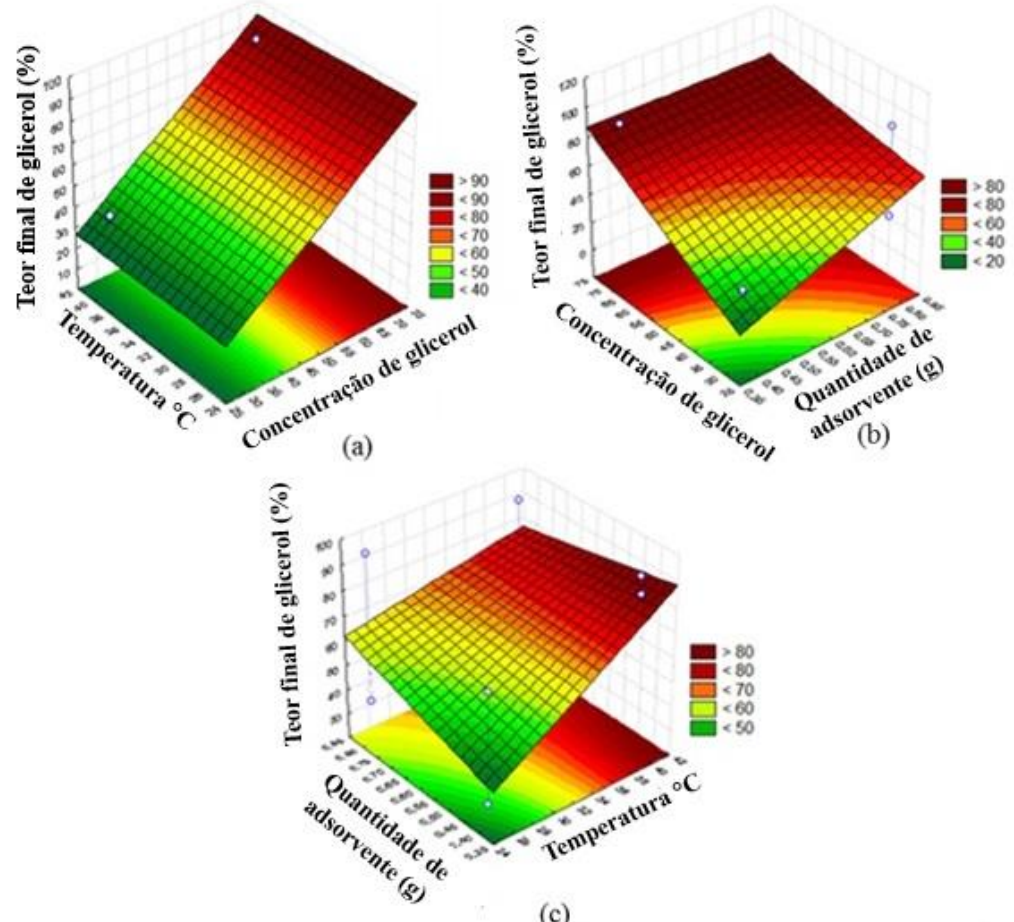

(c) 
Observa-se, pela Figura 1, que o teor final de glicerol varia diferentemente de um adsorvente para outro e, por isso, as superfícies de resposta foram apresentadas em função de diferentes variáveis significativas.

\section{CONCLUSÕES}

A partir da análise do planejamento experimental, pode-se concluir que os três adsorventes foram efetivos na remoção de óleo de soja da solução sintética utilizada, podendo assim ser utilizados no tratamento de glicerol oriundo da produção de biodiesel. Foram obtidos percentuais máximos de remoção $95,59 \%$, 97,25\% e 90,26\% utilizando carvão ativado, terra diatomácea e argila clarificante, respectivamente. Observa-se também que a variável mais significativa na remoção de contaminantes é a concentração inicial de glicerol na solução, tendo em vista que maiores percentuais de remoção foram obtidos utilizando concentrações iniciais de glicerol maiores.

\section{REFERÊNCIAS BIBLIOGRÁFICAS}

ALVES, Amanda de Paula. Purificação de glicerol utilizando diferentes adsorventes: Análise técnica e termodinâmica. 2017. 120p Dissertação (Mestrado em Engenharia Química, Tecnologia Química). Instituto de Tecnologia, Departamento de Engenharia Química, Universidade Federal Rural do Rio de Janeiro, Seropédica, RJ, 2017.

ANITHA, M.; KAMARUDIN S.K.; KOFLI, N.T. The potential of glycerol as a value-added commodity. Chemical Engineering Journal. v. 295, p. 119-130. 2016.

CHUN-HUI, Clayton Z.; BELTRAMINI, Jorge N.; FAN, Yong-Xian; LU, Max G. Q. Chemoselective Catalytic Conversion of Glycerol as a Biorenewable Source to Valuable Commodity Chemicals. Chemical Society Reviews. v. 37, p. 527-549. 2008.

DASARI, M. A.; KIATSIMKUL, P. P.; SUTTERLIN, W. R.; SUPPES, G. J.; Low-pressure hydrogenolysis of glycerol to propylene glycol. Applied Catalysis A: General. p.281, 225. 2005.

SILVA, G. P.; MACK, M.; CONTIERO, J., Glycerol: A promising and abundant carbon source for industrial microbiology. Biotechnology advances. v. 27, p.3 0-39. 2009.

SDRULA N. A study using classical or membrane separation in the biodiesel process. Desalination. v.50, p.1070-2. 2010. 